\title{
Optimization performance of irreversible refrigerators base on evolutionary algorithm
}

\author{
Mohammad Ali Ahmadi ${ }^{1}$, Milad Ashouri $^{2}$, Seyed Abbas Sadatsakkak $^{3}$ \\ and Mohammad H. Ahmadi ${ }^{4, a}$ \\ 1 Department of Petroleum Engineering, Ahwaz Faculty of Petroleum Engineering, Petroleum University \\ of Technology (PUT), Ahwaz, Iran \\ 2 Mechanical Engineering, Faculty of Mechanical Engineering, K. N. Toosi Univ. of Technology, No. 15-19, Pardis St., \\ Mollasadra Ave., Vanak Sq., Tehran 1999 143344, Iran \\ 3 Imam Khomeini International University, Qazvin, Iran \\ 4 Department of Mechanical Engineering, Pardis Branch, Islamic Azad University, Pardis New City, Tehran, Iran
}

Received 25 January 2015, Accepted 19 June 2015

\begin{abstract}
In early works done by authors, performance analysis of refrigeration systems such as power input, refrigeration load and coefficient of performance (COP) was investigated. In this article a new function called "Coefficient of Performance Exergy" or COPE has been introduced. Two objective functions of coefficient of performance exergy and exergy destruction are optimized simultaneously using the multiobjective optimization algorithm NSGAII. COPE has been maximized and exergy destruction has been minimized in order to get the best performance. Decision making has been done by means of two methods of LINAMP and TOPSIS. Finally an error analysis done for optimized values shows that LINAMP method is preferable against TOPSIS method.
\end{abstract}

Key words: Refrigeration / coefficient of performance / exergy destruction / decision making

\section{Introduction}

Numerous researches performed in finite-time thermodynamics where the coefficient of performance (COP) is selected as the objective function in the optimization analysis [1-4]. Performance optimization of the heat engines had been studied since 1996 by selection of the power density as the objective function [5-7] which is able to optimize the cycle performance containing the effects of the engine size. Similar study accomplished for the Ericsson [8] and Stirling [9] refrigeration cycles in which both the internal and external losses were neglected and the cooling load density was utilized as the optimization objective. Recently, significant strides have been made in the research and development for Brayton refrigeration cycles [10].

Numerous studies have been done since the 1970s for refrigerators to classify the performance restrictions and to optimize the thermodynamic cycles [11-22]. Most of the above mentioned work have chosen the input power, cooling load, exergy output rate, COP and entropy gen-

\footnotetext{
${ }^{a}$ Corresponding author:

mohammadhosein.ahmadi@gmail.com
}

eration rate as the optimization objectives. An ecological objective function for finite-time Carnot heat engines was first introduced by Angulo-Brown et al. [23] as $E^{\prime}=P-T_{L} S$ in which $T_{L}$ stands for the cold heat source temperature, $P$ is the output power and $\sigma$ represents the rate of entropy generation. Yan [24] improved this objective to $E=P-T_{0} S$ where $T_{0}$ is the ambient temperature.

Resolving multi-objective optimization issues is a complicated job for the reason that the subsequent various objective functions must be fulfilled at the same time [25]. By employing Evolutionary algorithms (EA) method, a multi-objective issue provides rise to a group of optimal solutions, each of the objective functions is fulfilled at a reasonable degree where the other answers are not being overshadowed [26]. On the whole, multi-objective optimization reveal an uncountable group of conceivable solutions named Pareto frontier. Today, multi-objective optimization of different systems in industries and energy engineering is engendering attention in numerous scholars in four corners of the world [27-44].

In the current work irreversible refrigerators were optimized using evolutionary algorithm while the coefficient of performance of exergy, the rate of exergy destruction 


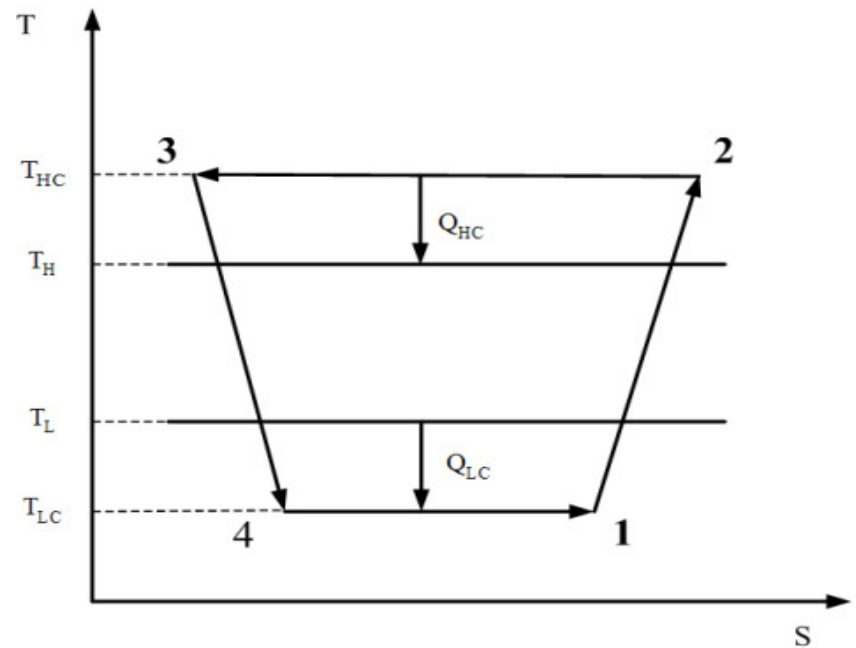

Fig. 1. $T-S$ diagram for the generalized refrigerator model.

are presumed as objectives of the optimization, while thermal operating variables of refrigerator including the internal irreversibility parameter $(\phi)$, the internal conductance of the refrigerator $(C)$, working fluid in the cycle works at temperature $T_{L C}$ and heat transfer surface area ratio $(f)$ are considered as decision variables.

\section{Model and basic assumption}

Figure 1 illustrates the temperature-entropy $(T-S)$ schematic diagram for an irreversible refrigerator. The temperatures of the heat source and heat sink where the cycle operates are represented by $T_{H}$ and $T_{L}$, correspondingly. The working fluid throughout the cycle operates at temperature $T_{L C}$ and $T_{H C}$, correspondingly. The temperature gradient $\left(T_{H C}-T_{H}\right)$ throughout the hightemperature heat exchanger creates $Q_{H C}$ while $Q_{L C}$ is made because to the driving force of $\left(T_{L}-T_{L C}\right)$. denotes the net heat transfer rate from the heat sink, viz., the cooling load $(R)$ and $Q_{H}$ stands for the net heat transfer rate to the heat source. The correlation between $T_{H}, T_{H C}$, $T_{L C}, T_{L}$ should satisfy the below expression

$$
T_{H C}>T_{H}>T_{L}>T_{L C}
$$

Figure 2 depicts a model used in the current paper for a universal irreversible refrigerator and its surrounds.

The model is based on some assumptions as follows:

(1) The steady state fluid flow is assumed for the working fluid and the cycle comprises of four irreversible processes including two adiabatic and two isothermal.

(2) The low-and high- temperature heat exchangers have finite heat transfer surface areas denoted by $F_{2}$ and $F_{1}$, respectively while the overall heat transfer surface area $(F)$ for the two aforementioned heat exchangers is presumed to be consistent:

$$
F=F_{1}+F_{2}
$$

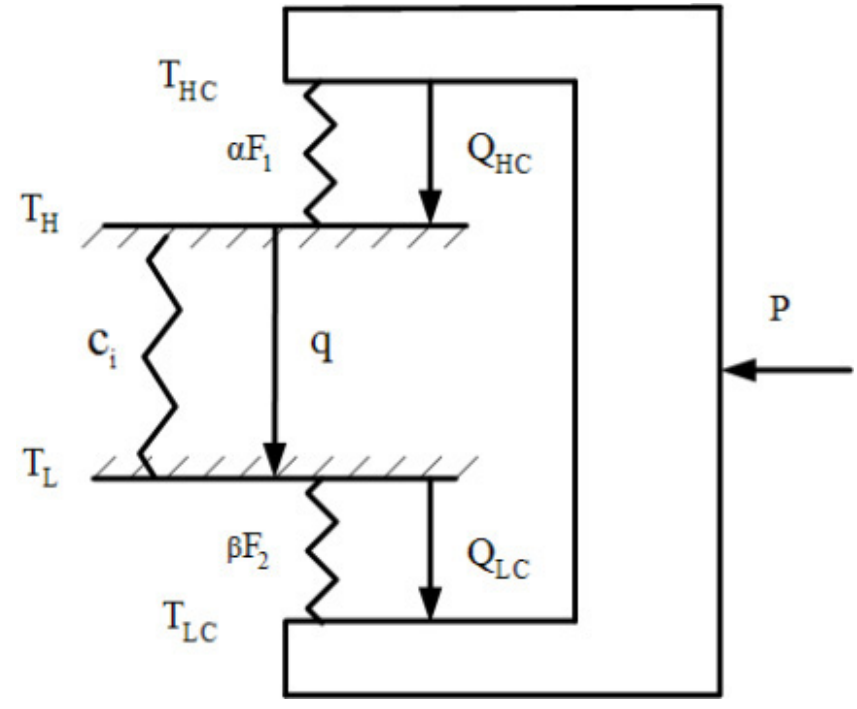

Fig. 2. Model of the generalized refrigerator and its surroundings.

(3) Due to the existence of heat leakage $(q)$ from the heat sink to the heat source, it is obtained as follows:

$$
\begin{aligned}
Q_{H} & =Q_{H C}-q \\
Q_{L} & =Q_{L C}-q=R \\
q & =C\left(T_{H}-T_{L}\right)
\end{aligned}
$$

(4) The irreversibilities throughout the cycle take place owing to: (i) thermal resistivity between the working fluid and the heat resources. (ii) heat loss among the heat resources and (iii) various parameters such as instability, friction and non-equilibrium accomplishments in the bounds of the refrigerator. Consequently, more power is required as input associated to an endoreversible refrigerator. The heat rejection rate to the heat sink $\left(Q_{H C}\right)$ of a universal irreversible refrigerator is much more than an endoreversible one $\left(Q_{H C}^{\prime}\right)$. These irreversibilities can be scaled by introducing a constant factor, $\phi$, which characterizes the extra internal varied irreversibility influence:

$$
\phi=\frac{Q_{H C}}{Q_{H C}^{\prime}} \geq 1
$$

Compared to the endoreversible [45] and irreversible [46-49] refrigerator approaches, the developed model is more general and reliable. If $q=0$ and $\phi=1$, the approach would be summary to the endoreversible refrigerator [45] while for $q>0$ and $\phi=1$, the approach is summary to an irreversible refrigerator with heat leak losses and heat resistance [46]. For $q=0$ and $\phi>1$, the approach is summary to the irreversible refrigerator with internal irreversibilities and heat resistance [46-49].

For an irreversible refrigerator, the second law of thermodynamics needs that:

$$
\frac{Q_{H C}^{\prime}}{Q_{L C}}=\frac{T_{H C}}{T_{L C}}
$$


Merging formulas (6) and (7) provides:

$$
\frac{Q_{H C}}{Q_{L C}}=\phi\left(\frac{T_{H C}}{T_{L C}}\right)
$$

We presume that the heat transfers among the refrigerator and its surrounds obey Newton's linear law:

$$
\begin{array}{r}
Q_{H C}=\alpha F_{1}\left(T_{H C}-T_{H}\right) \\
Q_{L C}=\beta F_{2}\left(T_{L}-T_{L C}\right)
\end{array}
$$

Moreover, following formula defines a heat transfer surface area ratio $(f)$ :

$$
f=\frac{F_{1}}{F_{2}}
$$

According to the first law of thermodynamics, the power input $(P)$ to the refrigerator can be determined via following equation:

$$
P=Q_{H C}-Q_{L C}=Q_{H}-Q_{L}=Q_{H}-R
$$

The coefficient of performance $(C O P)$ of the refrigerator is:

$$
C O P=\frac{Q_{L}}{P}=\frac{R}{P}
$$

Equations (7)-(13) provide:

$$
\begin{aligned}
C O P & =\frac{R}{(R+q)\left(\phi\left(\frac{T_{H C}}{T_{L C}}\right)-1\right)} \\
S & =\frac{Q_{H}}{T_{H}}-\frac{Q_{L}}{T_{L}}
\end{aligned}
$$

Merging Equations (8)-(11) gives:

$$
\frac{Q_{H C}}{R+q}=\phi\left(\frac{T_{H C}}{T_{L C}}\right)=\frac{f \alpha\left(T_{H C}-T_{H}\right)}{\beta F_{2}\left(T_{L}-T_{L C}\right)}
$$

which then yields:

$$
\frac{T_{H C}}{T_{L C}}=\frac{T_{H}(f \alpha / \beta)}{(\phi+f \alpha / \beta) T_{L C}-\phi T_{L}}
$$

Merging Equations (2) and (9)-(11) provides:

$$
T_{L C}=T_{L}-\frac{(R+q)(1+f)}{\beta F}
$$

Replacing formula (18) into formulas (16) and (17), then following equations can be obtained:

$$
\begin{aligned}
\frac{T_{H C}}{T_{L C}} & =\frac{T_{H}(f \alpha / \beta)}{(\phi+f \alpha / \beta)\left(T_{L}-\frac{(R+q)(1+f)}{\beta F}\right)-\phi T_{L}} \\
Q_{H C} & =\frac{T_{H} \phi(R+q)}{T_{L}-(R+q)(1+f) \frac{\left(\frac{\phi}{\alpha}+\frac{f}{\beta}\right)}{f F}}
\end{aligned}
$$

To derive the entropy generation rate $(S)$ and coefficient of performance $(C O P)$ of the generalized irreversible refrigerator cycle, we substitute Equations (19) and (20) into Equations (14) and (15) as following:

$$
\begin{gathered}
S=\frac{\phi(R+q)}{T_{L}-(R+q)(1+f) \frac{\left(\frac{\phi}{\alpha}+\frac{f}{\beta}\right)}{f F}}-\frac{R}{T_{L}}-\frac{q}{T_{H}} \\
C O P=\left[\frac{R}{R+q}\right]\left\{\frac{\phi T_{H}}{\left(T_{L}-(R+q)(1+f) \frac{\left(\frac{\phi}{\alpha}+\frac{f}{\beta}\right)}{f F}-1\right)}\right\}^{-1}
\end{gathered}
$$

From exergy analysis point of view, the objective function of ecological optimization, suggested by AnguloBrown [23] and improved by Yan [24], can be obtained via following equation:

$$
E=P-T_{0} S
$$

in which $T$ denotes the temperature of environment.

The ecological coefficient of performance $(E C O P)$ was proposed by Ust and colleagues [22], as the proportion of power output to the loss rate of availability, i.e.

$$
E C O P=\frac{P}{T_{0} S}
$$

From exergy analysis point of view, Chen and colleagues [50] present an ecological optimization objective for refrigerator cycles as following:

$$
\begin{aligned}
\dot{I} & =T_{0} S \\
E & =R\left[\left(\frac{T_{0}}{T_{L}}-1\right)-\left(1+\frac{1}{C O P}\right)\left(\frac{T_{0}}{T_{H}}-1\right)\right]-T_{0} S
\end{aligned}
$$

The coefficient of performance of exergy $(C O P E)$ is proposed as the proportion of exergy loss rate (entropy generation rate) and the exergy output rate, consequently, $C O P E$ is a dimensionless ecological function and can be written as following equation:

$$
C O P E=\frac{R\left[\left(\frac{T_{0}}{T_{L}}-1\right)-\left(1+\frac{1}{C O P}\right)\left(\frac{T_{0}}{T_{H}}-1\right)\right]}{T_{0} S}
$$

\section{Multi-objective optimization with evolutionary algorithms}

\subsection{Optimization via EA}

Using genetic algorithm (GA) which is classified under evolutionary algorithms, we obtained Pareto frontier. John Holland was the first who suggested and developed genetics algorithm in the 1960s which integrates natural adaptation approach with computer algorithms and numerical optimization techniques $[25,26]$. A computer 


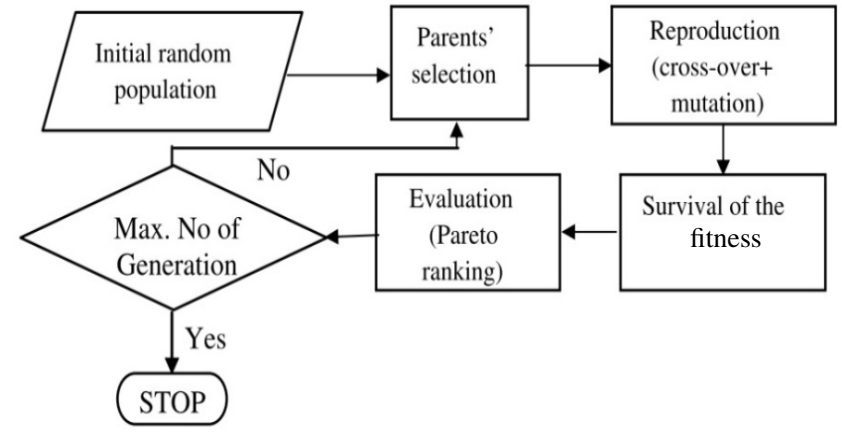

Fig. 3. Scheme for the multi-objective evolutionary algorithm used in the present study [28-33].

simulation is used for optimization problem and generation of acceptable solution where a population of abstract demonstrations named chromosomes of nominee answers named individuals evolves. The random population of generated individuals is the start point of the evolution and the generation process repeats. In every stage, the assessment of each individual fitness is executed and multiple individuals are chosen arbitrarily from the present population. Next, they adapted and lastly a fresh population is produced. Each generated population is needed to be used for the next step of the algorithm $[25,26]$. The complication of conventional approaches can be condensed by multi-objective evolutionary algorithms (MOEAs) which have recently been progressive by employing various examinations on math based problems $[25,26]$. Figure 3 demonstrates the schematic of the MOEA [28-33].

\subsection{Objective functions, decision parameters and limitations}

Two important objective functions for optimization are the exergy destruction (should be minimized), the coefficient of performance of exergy (should be maximized) represented by Equations (24) and (26), correspondingly.

Throughout this research, four decision parameters are presumed as following:

- $\phi$ internal irreversibility parameter.

- $C$ the internal conductance of the refrigerator.

- $f$ the heat transfer surface area ratio.

$-T_{L C}$ working fluid temperature $(\mathrm{K})$.

The objective functions in regard to below limitations are unraveled:

$$
\begin{aligned}
& 0.01 \leq C \leq 0.03(\mathrm{~kW} / \mathrm{K}) \\
& 1 \leq \phi \leq 1.3 \\
& 0.5 \leq f \leq 4 \\
& 240 \leq T_{L C} \leq 255
\end{aligned}
$$

\subsection{Decision-making in the multi-objective optimization}

Next to the multi-parameters and objectives optimization process, picking a final optimal answer from the solutions achieved by MOEA has significant status. In this regard, various approaches which recognized as decision makers can be employ to conclude desire optimum parameters from the Pareto frontier which is formerly achieved. In this paper, two well-known, effective and rapid decision makers containing LINMAP and TOPSIS methods are employed. Final optimal answers were decided based on the expertise and criteria which proposed by each decision maker. Explanations of these decision makers are demonstrated in references [28-33].

\section{Result and discussion}

The coefficient of performance of exergy $(C O P E)$ is maximized simultaneously and the exergy destruction $\left(T_{0} S\right)$ is minimized concurrently employing the multiobjective optimizing approach which operates according to the NSGA-II method.

By the way, optimization is accomplished via objective functions that are formulated by Equations (25) and (27) limitations which are represented via Equations $(28)-(31)$.

With the intention of have reliability with earlier publications, descriptions of the irreversible refrigerator cycle are presumed as following as [51],

$$
T_{H}=300 \mathrm{~K}, T_{L}=260 \mathrm{~K}, T_{0}=290 \mathrm{~K}
$$

Pareto optimal frontier is exhibited in Figure 4 and also, obtained optimum solutions of LINMAP and TOPSIS methods are exhibited in Figure 4. From Figure 4 it can be seen that optimal solution of COPE varied of 3.3 to 3.5 and optimal solution of $T_{0} S$ varied of 0.17 to 0.24 .

Figures 5 to 8 exhibit the distribution of different values of decision parameter in their permissible range for the optimum design points on the Pareto front. It can be seen from Figure 5 that distribution of $C$ in $C=0.01$ was marked by blue line and $C$ obtained lower value. From Figure 6 it can be seen that distribution of $\phi$ in $\phi=1$ was marked by blue line and $\phi$ obtained lower value. From Figure 7 it can be seen that distribution of various values of $f$ with the range of 2.48 to 4 was further. It can be seen from Figure 8 that distribution of $T_{L C}$ in $T_{L C}=255 \mathrm{~K}$ was marked by blue line and $T_{L C}$ obtained higher value.

Table 1 reports optimum solutions gained throughout this research employing two decision making approaches.

\subsection{Error analysis}

For error analysis, the mean absolute percentage error (MAPE) is employed. For this goal, 30 runs of each method are accomplished to provide ultimate outcome. First and second row of Table 2 show maximum absolute percentage error (MAAE) and (MAPE) respectively. 
M.A. Ahmadi et al.: Mechanics \& Industry 17, 209 (2016)

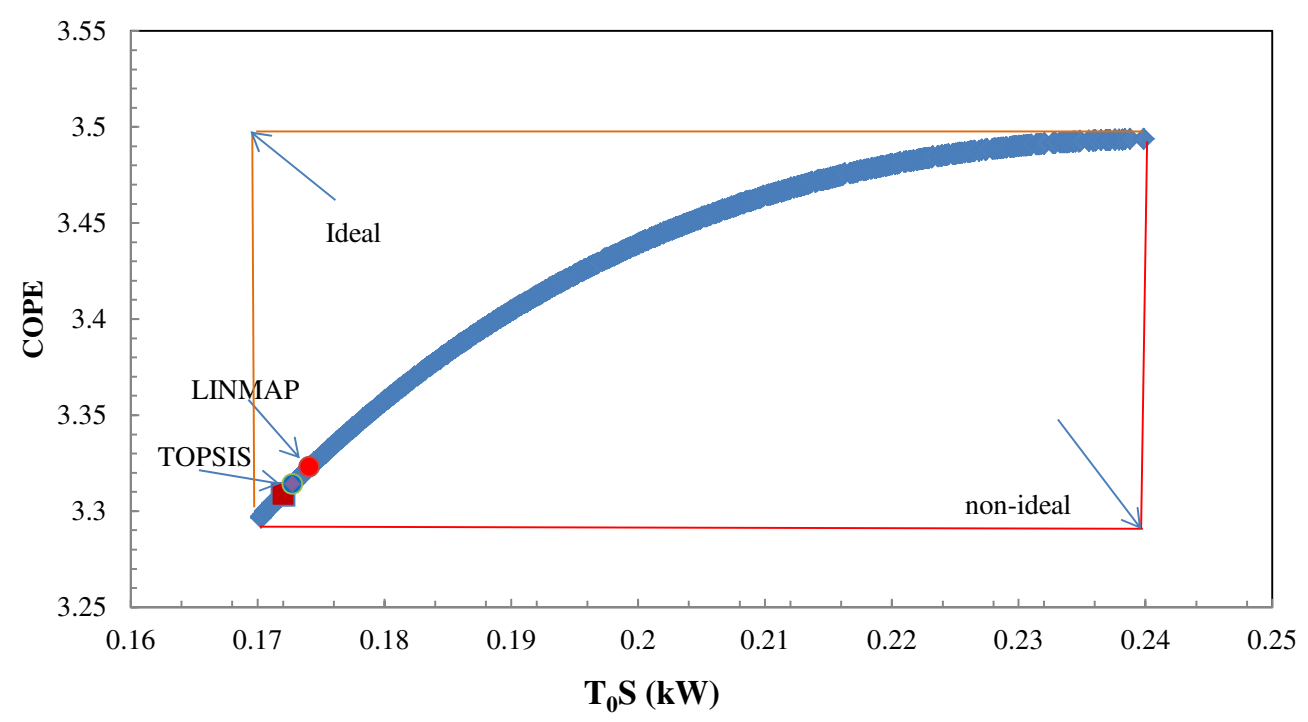

Fig. 4. Pareto frontier (Pareto optimal solutions) for $T_{0} S$ versus COPE using NSGA-II.

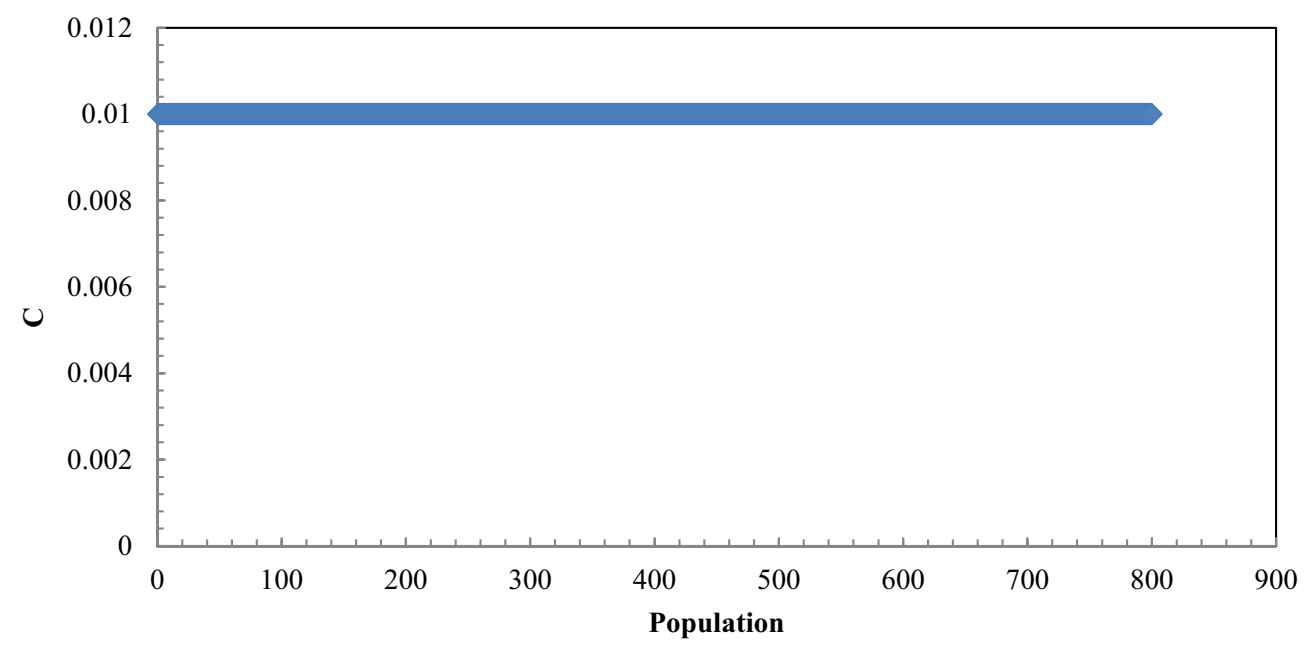

Fig. 5. Distribution of $C$ for the optimal points on Pareto front.

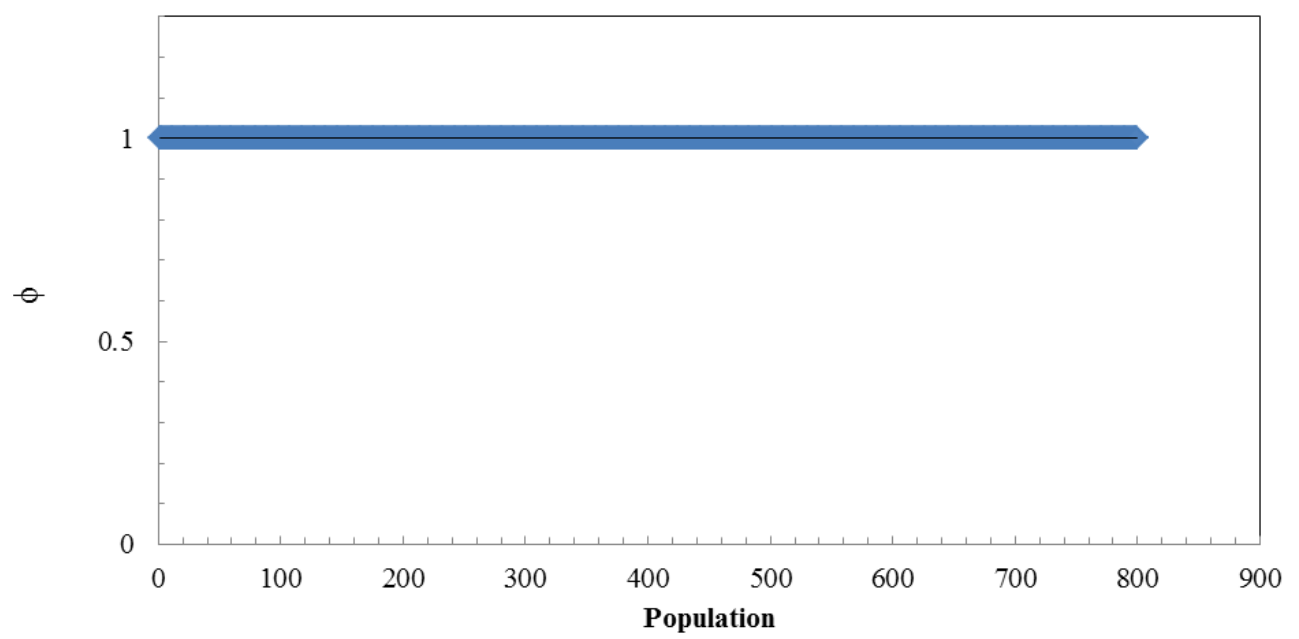

Fig. 6. Distribution of $\phi$ for the optimal points on Pareto front. 
M.A. Ahmadi et al.: Mechanics \& Industry 17, 209 (2016)

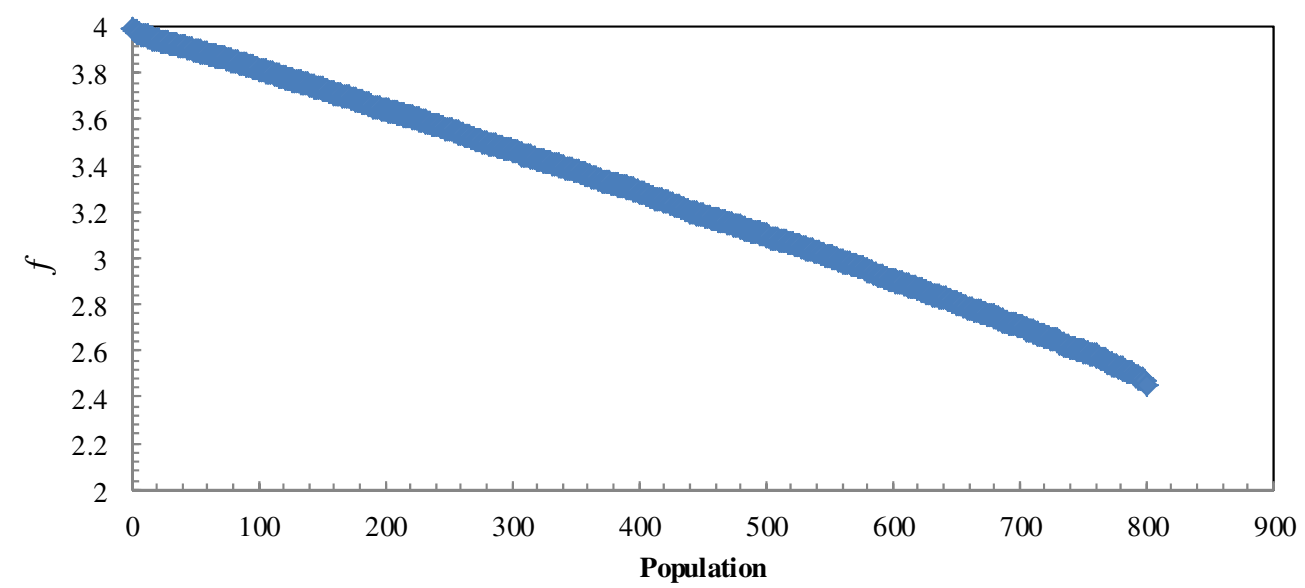

Fig. 7. Distribution of $f$ for the optimal points on Pareto front.

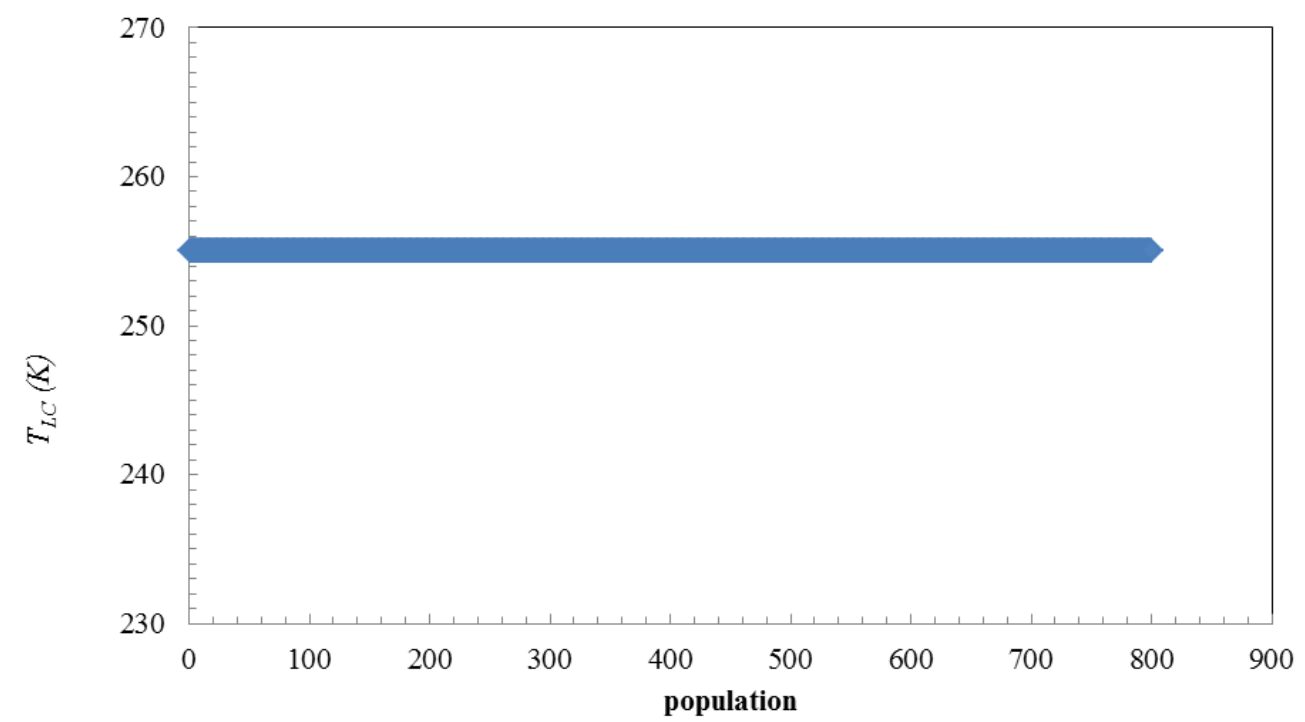

Fig. 8. Distribution of $\phi$ for the optimal points on Pareto front.

Table 1. Decision making of multi-objective optimal solutions.

\begin{tabular}{ccccccc}
\hline \multirow{2}{*}{ Decision Making Method } & \multicolumn{3}{c}{ Decision variables } & \multicolumn{3}{c}{ Objective functions } \\
\cline { 2 - 7 } & $C$ & $\phi$ & $f$ & $T_{L C}$ & $T_{0} S$ & $C O P E$ \\
\hline TOPSIS & 0.01 & 1.000013 & 3.90 & 255 & 0.17272 & 3.3142 \\
LINMAP & 0.01 & 1.000013 & 3.85 & 255 & 0.17408 & 3.3232 \\
\hline
\end{tabular}

Table 2. Error analysis based on the mean absolute percent error (MAPE) method.

\begin{tabular}{ccccc}
\hline Decision making method & \multicolumn{2}{c}{ TOPSIS } & \multicolumn{2}{c}{ LINMAP } \\
\hline Objectives & $C O P E$ & $T_{0} S$ & $C O P E$ & $T_{0} S$ \\
\hline Max Error \% & 4.13 & 14.86 & 3.88 & 15.04 \\
Average Error $\%$ & 1.74 & 5.43 & 1.64 & 5.41 \\
\hline
\end{tabular}




\section{Conclusions}

In this study, thermodynamic analysis has been applied to determine the exergy destruction and the coefficient of performance of exergy $(C O P E)$ of the refrigerator. The exergy destruction and the COPE of the refrigerator are presumed concurrently for multi-objective optimization where the internal irreversibility parameter $(\phi)$, the internal conductance of the refrigerator $(C)$, heat transfer surface area ratio $(f)$ and working fluid in the cycle operating at temperature $T_{L C}$ are presumed as design variables. Multi objective evolutionary approach is presumed according to the NSGA-II method and the Pareto optimal frontier throughout objectives space is acquired. An ultimate optimum answer is nominated from answers of the Pareto frontier employing two decision making approaches comprising TOPSIS and LINMAP techniques.

Acknowledgements. This paper was originally presented in 1st International Electronic Conference on Entropy and Its Applications.

\section{References}

[1] A. Bejan, Entropy Generation Minimization, CRC Press, Boca Raton FL, 1996

[2] R.S. Berry, V.A. Kazakov, S. Sieniutycz, Z. Szwast, A.M. Tsirlin, Thermodynamic Optimization of Finite Time Processes, Wiley, Chichester, 1999

[3] L. Chen, C. Wu, F. Sun, Finite time thermodynamics optimization or entropy generation minimization of energy systems, J. Non-Equilibrium Thermodyn. 24 (1999) 327

[4] C. Wu, L. Chen, J. Chen, (eds.), Recent Advances in Finite Time Thermodynamics, Nova Science Publishers, New York, 1999

[5] B. Sahin, A. Kodal, H. Yavuz, Maximum power density analysis of an endoreversible Carnot heat engine, Energy, Int. 21 (1996) 1219

[6] L. Berrin, A. Sisman, H. Yavuz, Analysis of Ericsson cycle at maximum power density conditions, ECOS 1996, pp. $25-27$

[7] L. Chen, J. Lin, F. Sun, C. Wu, Efficiency of an Atkinson engine at maximum power density, Energy Convers. Mgmt. 39 (1998) 337

[8] H. L. Yavuz, L.B. Erbay, General performance characteristics of an Ericsson refrigerator, ECOS 1998, Nancy, France, pp. 565-571

[9] L.B. Erbayan d H. Yavuz, The maximum cooling density of a realistic Stirling refrigerator, J. Phys. D. 31 (1998) 291

[10] J.A. Mc Cormick, Progress on the development of miniature turbo-machines for low capacity reverse Brayton cryocooler, Proc. 9-th Int. Cryocooler Conf., 1996

[11] L.G. Chen, C. Wu, F.R. Sun, Finite time thermodynamic optimization or entropy generation minimization of energy system, J. Non-Equil. Thermodyn. 24 (2005) $327-359$

[12] L.G. Chen, L. Zhang, F.R. Sun, Power, efficiency, entropy-generation rate and ecological Optimization for a class of generalized irreversible universal heat-engine cycles, Appl. Energy 84 (2007) 512-525
[13] A. Durmayaz, O.S. Sogut, B. Sahin, H. Yavuz, Optimization of thermal systems based on finite-time thermodynamics and thermoeconomics, Prog. Energy Combust. Sci. 30 (2004) 175-21

[14] C.K. Chen, Y.F. Su, Exergetic efficiency optimization for an irreversible Brayton refrigeration cycle, Int. J. Therm. Sci. 44 (2005) 303-310

[15] Y. Tu, L.G. Chen, F.R. Sun, C. Wu, Cooling load and efficient of performance optimizations for real airrefrigerators, Appl. Energy 83 (2006) 1289-1306

[16] X.Q. Zhu, L.G. Chen, F.R. Sun, C. Wu, Effect of heat transfer law on the ecological optimization of a generalized irreversible Carnot heat pump, Int. J. Exergy 2 (2005) 423-436

[17] L.G. Chen, X.Q. Zhu, F.R. Sun, C. Wu, Ecological optimization of a generalized irreversible Carnot refrigerator for a generalized heat transfer law, Int. J. Ambient Energy 28 (2007) 213-219

[18] X.Q. Zhu, L.G. Chen, F.R. Sun, C. Wu, Exergy based ecological optimization for a generalized irreversible Carnot refrigerator, J. Energy Inst. 79 (2006) 42-46

[19] L.G. Chen, J. Li, F.R. Sun, Generalized irreversible heatengine experiencing a complex heat-transfer law, Appl. Energy 85 (2008) 52-60

[20] J. Chen, X. Chen, C. Wu, Optimization of rate of exergy output of a multistage endoreversible combined refrigeration system, Exergy 1 (2001) 100-106

[21] T. Morosuk, G. Tsatsaronis, Advanced exergetic evaluation of refrigeration machines using different working fluids, Energy 34 (2009) 2248-2258

[22] Y. Ust, Performance analysis and optimization of irreversible air refrigeration cycles based on ecological coefficient of performance criterion, Appl. Therm. Eng. 29 (2009) 47-55

[23] F. Angulo-Brown, An ecological optimization criterion for finite-time heat engines, J. Appl. Phys. 69 (1991) 7465-7469

[24] Z. Yan, Comment on ecological optimization criterion for finite-time heat-engines, J. Appl. Phys. 73 (1993) 3583

[25] D.A.V. Veldhuizen, G.B. Lamont, Multi-objective Evolutionary Algorithms: Analyzing the State-of-theArt, Evolutionary Computation 8 (2000) 125-147

[26] A. Konak, D.W. Coit, A.E. Smith, Multi-objective optimization using genetic algorithms: A tutorial, Reliability Engineering \& System Safety 91 (2006) 992-1007

[27] T. Bck, D. Fogel, Z. Michalewicz, Handbook of evolutionary computation, Oxford Univ., Press, 1997

[28] M.H. Ahmadi, H. Hosseinzade, H. Sayyaadi, A.H. Mohammadi, F. Kimiaghalam, Application of the multiobjective optimization method for designing a powered Stirling heat engine: design with maximized power, thermal efficiency and minimized pressure loss, Renew. Energy 60 (2013) 313-22

[29] M.H. Ahmadi, H. Sayyaadi, A.H. Mohammadi, A. Marco, Barranco-Jimenez. Thermo-economic multi-objective optimization of solar dish-Stirling engine by implementing evolutionary algorithm, Energy Convers. Manag. 73 (2013) 370-380

[30] M.H. Ahmadi, M.A. Ahmadi, A.H. Mohammadi, M. Mehrpooya, M. Feidt, Thermodynamic optimization of Stirling heat pump based on multiple criteria, Energy Convers. Manag. 80 (2014) 319-328 
[31] A. Lazzaretto, A. Toffolo, Energy, economy and environment as objectives in multi-criterion optimization of thermal systems design, Energy 29 (2004) 1139-1157

[32] M.H. Ahmadi, M.A. Ahmadi, A.H. Mohammadi, M. Feidt, S.M. Pourkiaei, Multi-objective optimization of an irreversible Stirling cryogenic refrigerator cycle, Energy Convers. Manage. 82 (2014) 351-360

[33] M.H. Ahmadi, A.H. Mohammadi, S.Dehghani, Evaluation of the maximized power of a regenerative endoreversible Stirling cycle using the thermodynamic analysis, Energy Convers. Manage. 76 (2013) 561-570

[34] S. Toghyani, A. Kasaeian, M.H. Ahmadi, Multi-objective optimization of Stirling engine using non-ideal adiabatic method, Energy Convers. Manage. 80 (2014) 54-62

[35] H. Sayyaadi, M.H. Ahmadi, S. Dehghani, Optimal Design of a Solar-Driven Heat Engine Based on Thermal and Ecological Criteria, J. Energy Eng. (2014), DOI: 10.1061/(ASCE)EY.1943-7897.0000191, 04014012

[36] H. Sahraie, M.R. Mirani, M.H. Ahmadi, M. Ashouri, Thermo-economic and thermodynamic analysis and optimization of a two-stage irreversible heat pump, Energy Convers. Manage. 99 (2015) 81-91

[37] M.H. Ahmadi, M.A. Ahmadi, M. Mehrpooya, H. Hosseinzade, M. Feidt, Thermodynamic and thermoeconomic analysis and optimization of performance of irreversible four-temperature-level absorption refrigeration, Energy Convers. Manage. 88 (2014) 1051-1059

[38] M.H. Ahmadi, M.A. Ahmadi, Thermodynamic analysis and optimization of an irreversible Ericsson cryogenic refrigerator cycle, Energy Convers. Manage. 89 (2015) $147-55$

[39] M.H. Ahmadi, M.A. Ahmadi, M. Mehrpooya, M. Sameti, Thermo-ecological analysis and optimization performance of an irreversible three-heat-source absorption heat pump, Energy Convers. Manage. 90 (2015) 175-183

[40] M.H. Ahmadi, M.A. Ahmadi, M. Feidt, Performance optimization of a solar-driven multi-step irreversible Brayton cycle based on a multi-objective genetic algorithm. Oil \& Gas Science and Technology - Rev. IFP Energies nouvelles 2014. http://dx.doi.org/10.2516/ ogst/2014028
[41] M.H. Ahmadi, M.A. Ahmadi, M. Feidt, Thermodynamic analysis and evolutionary algorithm based on multiobjective optimization of performance for irreversible four-temperature-level refrigeration, Mechanics \& Industry 16 (2015) 207

[42] S.A. Sadatsakkak, M.H. Ahmadi, M.A. Ahmadi, Thermodynamic and thermo-economic analysis and optimization of an irreversible regenerative closed Brayton cycle, Energy Convers. Manage. 94 (2015) 124-129

[43] S.A. Sadatsakkak, et al., Optimization density power and thermal efficiency of an endoreversible Braysson cycle by using non-dominated sorting genetic algorithm, Energy Convers. Manage. 93 (2015) 31-39

[44] S.A. Sadatsakkak, M.H. Ahmadi, M.A. Ahmadi, Optimization performance and thermodynamic analysis of an irreversible nano scale Brayton cycle operating with Maxwell-Boltzmann gas, Energy Convers. Manage. 101 (2015) 592-605

[45] L.G. Chen, F.R. Sun, C. Wu, Ecological optimization criteria for an endoreversible Carnot refrigerator (in Chinese), Nat. J. 15 (1992)

[46] J.M. Gordon, K.C. Ng, Thermodynamic modeling of reciprocating chillers, J. Appl. Phys. 75 (1994) 2769-2774

[47] F.R. Sun, C. Wu, L.G. Chen, Optimal performance and rate of entropy production for forward and reverse irreversible Carnot cycles, Chin. J. Eng. Thermophys. 12 (1991) 357-360

[48] M.M. Ait-Ali, A class of internally irreversible refrigeration cycles. J. Phys. D 29 (1996) 593-599

[49] M.M. Ait-Ali, The maximum coefficient of performance of internally irreversible refrigerators and heat pumps, J. Phys. D 29 (1996) 975-980

[50] L.G. Chen, X. Zhu, F.R. Sun, C. Wu, Ecological optimization for generalized irreversible Carnot refrigerators, J. Phys. D 35 (2005) 113-118

[51] J. Xu, L. Pang, J. Wang, Performance Optimization of Generalized Irreversible Refrigerator Based on a New Ecological Criterion, Entropy 15 (2013) 5277-5291 